\title{
STUDY ON BREAKUP OF LIQUID LIGAMENTS IN HYPERSONIC CROSS FLOW USING LASER SHEET IMAGING AND INFRARED LIGHT EXTINCTION SPECTROSCOPY
}

\author{
T. Regert ${ }^{1}$, I. Horvath ${ }^{1}$, J.-M. Buchlin ${ }^{1}$, D. Masutti ${ }^{1}$, \\ O. Chazot ${ }^{1}$, M. R. Vetrano ${ }^{1}$, C. Lapebie ${ }^{2}$, and C. Le Gallic ${ }^{2}$ \\ ${ }^{1}$ Von Karman Institute for Fluid Dynamic \\ Belgium \\ ${ }^{2}$ CEA Gramat \\ France
}

\begin{abstract}
This paper presents and discusses the results of tests of breakup phenomenon of liquid water into a hypersonic cross flow from the surface of a 7 degree half-angle cone model at zero degree angle of incidence. The present work shows the dependence of the liquid phase characteristics on the cross-section area of the injection hole in a Mach 6 cross flow. The results are analyzed qualitatively by imaging, by Interferometric Laser Imaging for Droplet Sizing (ILIDS), and by InfraRed Light Extinction Spectroscopy (IR-LES). Conclusions are drawn concerning the droplet size distribution and the liquid flow field characteristics.
\end{abstract}

\section{INTRODUCTION}

The aerospace and several diverse fields of the industry have the interest in understanding better the breakup of liquids in high Mach number flows leakage problems of high-speed vehicles that might influence the geometry of the vehicle due to ice deposition or interaction between the generated droplets and the aft surfaces of the vehicles; signal transmission problems due to the high-speed metal particles in supersonic jets; scramjet research applications; painting industry when producing fine metal powders from liquid metals exposed to high-speed gas flow, etc.

The present work shows the dependence of the droplet cloud characteristics on the cross-section area of the injection hole in a Mach 6 cross flow that influences the Weber number.

(C) The authors, published by EDP Sciences. This is an Open Access article distributed under the terms of the Creative Commons Attribution License 4.0 (http://creativecommons.org/licenses/by/4.0/). 
There are only few contributions in the scientific literature that deal with the fragmentation of liquids in high-speed compressible gas flows, although there are many for low speeds, i. e., for incompressible gas flows. The basic literature of breakup of droplets due to gas flow is the work [1]. A droplet exposed to a sudden impulse of flow at certain velocity leads to a wide variety of possible scenarios that can happen to the droplet which is represented in the very well known map of Pilch and Erdman [1] and depicted in Fig. 1. As the gas flow velocity is increasing relative to the droplet, the droplet breaks up into two twin droplets.
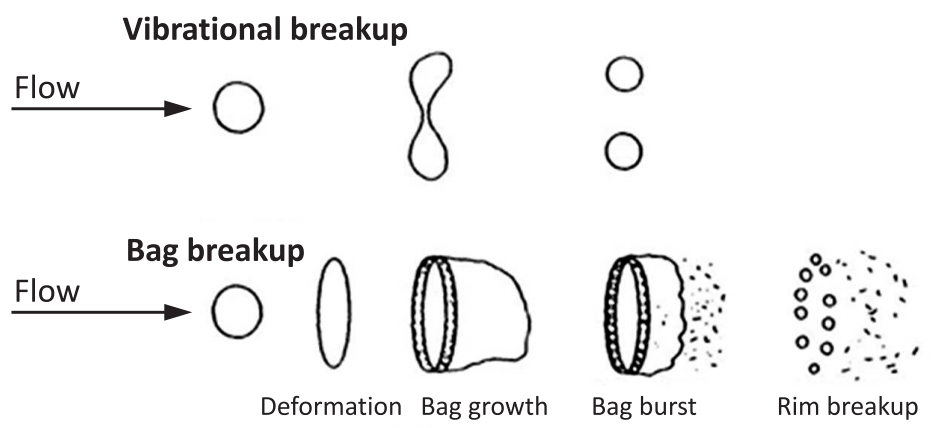

Bag-and-stamen breakup
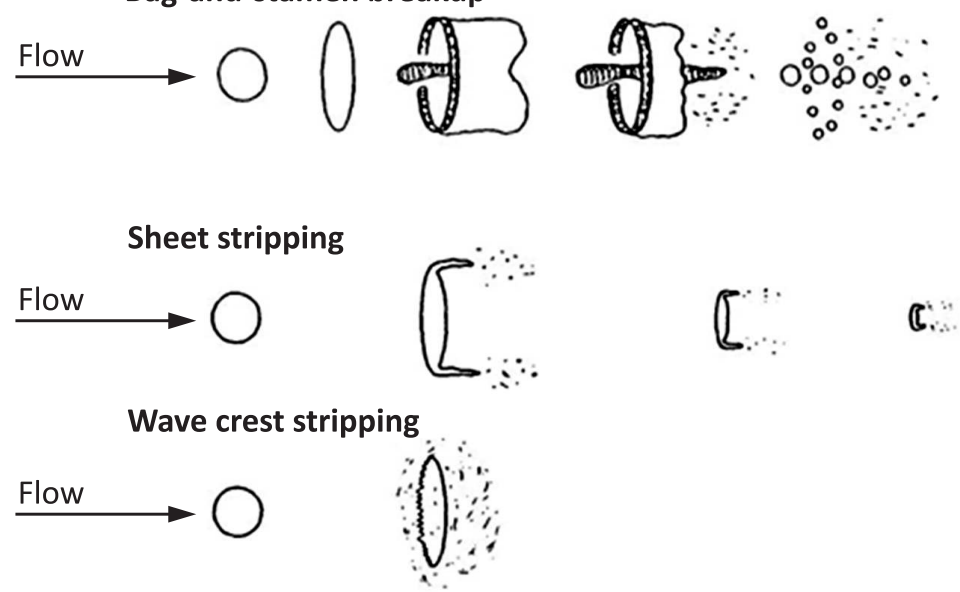

Catastrophic breakup

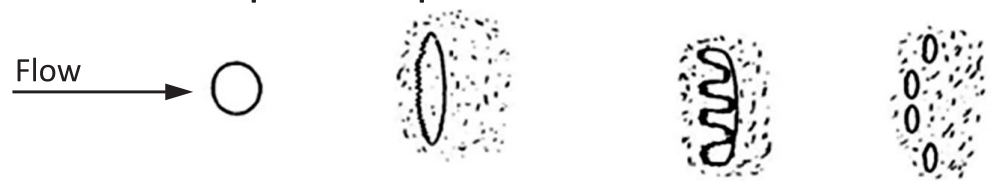

Figure 1 Breakup map according to Pilch and Erdman [1] 
At higher velocities, one encounters different forms of bag-type breakups, sheet stripping, wave crest stripping, and, finally, catastrophic breakup. The main nondimensional number that is associated with these types of breakup forms is the Weber number which is based on the relative momentum of the gas flow, the initial droplet diameter and the surface tension of the liquid:

$$
\mathrm{We}=\frac{d_{j} \rho_{\text {gas }}\left(U_{\text {gas }}-U_{\text {jet }}\right)^{2}}{\sigma}
$$

where $\rho_{\text {gas }}$ is the density of the gas, $\mathrm{kg} / \mathrm{m}^{3} ; U_{\text {gas }}$ is the velocity of the gas, $\mathrm{m} / \mathrm{s}$; $U_{\text {jet }}$ is the velocity of the liquid jet, $\mathrm{m} / \mathrm{s} ; d_{j}$ is the diameter of the jet, $\mathrm{m}$; and $\sigma$ is the surface tension of the liquid, $\mathrm{N} / \mathrm{m}$.

The other mentioned nondimensional number, the Ohnesorge number, expresses the ratio of the viscous forces to the inertial and the surface tension forces in the liquid body and is defined as

$$
\mathrm{Oh}=\frac{\mu_{j}}{\sqrt{\rho_{j} d_{j} \sigma}}
$$

where $\mu_{j}$ is the dynamic viscosity of the liquid, $\mathrm{Pa}$; and $\rho_{j}$ is the density of the liquid, $\mathrm{kg} / \mathrm{m}^{3}$.

Although the critical Weber numbers corresponding to the breakup mechanisms of the liquid body depend on the Ohnesorge number, its effect was not considered in the present project because of the very high magnitude of the Weber number $\left(10^{4}\right)$. According to the literature (see, for example, $\left.[1,2]\right)$, the minimum value of Weber number at where the droplet will not longer break up into smaller droplets, i. e., above which the formation of two twin droplets is expected, is around 12. The other end, i. e., where catastrophic breakup occurs, is above 350. In case of high-speed flows, more specifically above Mach 1, the Weber number is expected to be above 350 for many practical cases, unless the droplets or liquid ligaments are injected into the flow with very low relative velocity between the gas and liquid phase. An overview of liquid injection into compressible flow can be found in [3], jet penetration height into a compressible cross flow in the range of Mach 2 to Mach 4 was investigated in [4-8], and droplets were exposed to Mach 5 cross flow in a shock tube in [9]. A liquid jet in a Mach 6 cross flow over a flat plate was investigated in detail in [10]. Droplet size distribution measurements are rarely available and they were performed, mainly, by Phase Doppler Interferometry (PDI) or backlighting imaging. More recently, the ILIDS technique is gaining space in droplet size measurements as was applied for a spray of water injected downstream from the base of a model in Mach 6 flow [11]. These techniques have serious limitation concerning the droplet clouds with high droplet concentration.

A new method that has originally been developed for nanoparticles was tested in this work for the determination of the droplet sizes in a dense droplet cloud. 
This method is based on the multiwavelength light extinction technique. As the light wavelength for this technique has to be close or larger than the size of the droplets to be measured, in the present case, it has to be in the infrared range; thus, the technique is referred to infrared light extinction spectroscopy.

\section{FACILITY AND MODEL}

The VKI hypersonic tunnel H3 is an open loop blowdown facility supplied by high-pressure air-feeding axisymmetric nozzle that provides a Mach 6 free jet $12 \mathrm{~cm}$ in diameter. The test gas passes through a pebble-bed heater as illustrated in Fig. 2, which supplies air at temperature ranging from ambient to $550 \mathrm{~K}$ and pressures ranging from 7 to 35 bar. Unit Reynolds number may be varied from $9 \cdot 10^{6}$ to $30 \cdot 10^{6} 1 / \mathrm{m}$.

The test section contains a three-degree-of-freedom traversing system for model or probe support that also allows the angle of incidence to vary between $\pm 5^{\circ}$. A fast acting valve serves to rapidly open and close the high-pressure pipeline to prevent the problems when starting or stopping the flow field. To avoid wind-tunnel blockage, the facility is started without the model in the test section. A fast injection mechanism is used to place the model or probes in the stream once the hypersonic flow is established and stable. Within the axisymmetric nozzle, the flow is considered to be uniform. At a distance of $100 \mathrm{~mm}$ from the nozzle, the Mach number is found to vary between 5.92 and 6.07 in the furthest region from the center of the jet. The tunnel is equipped with

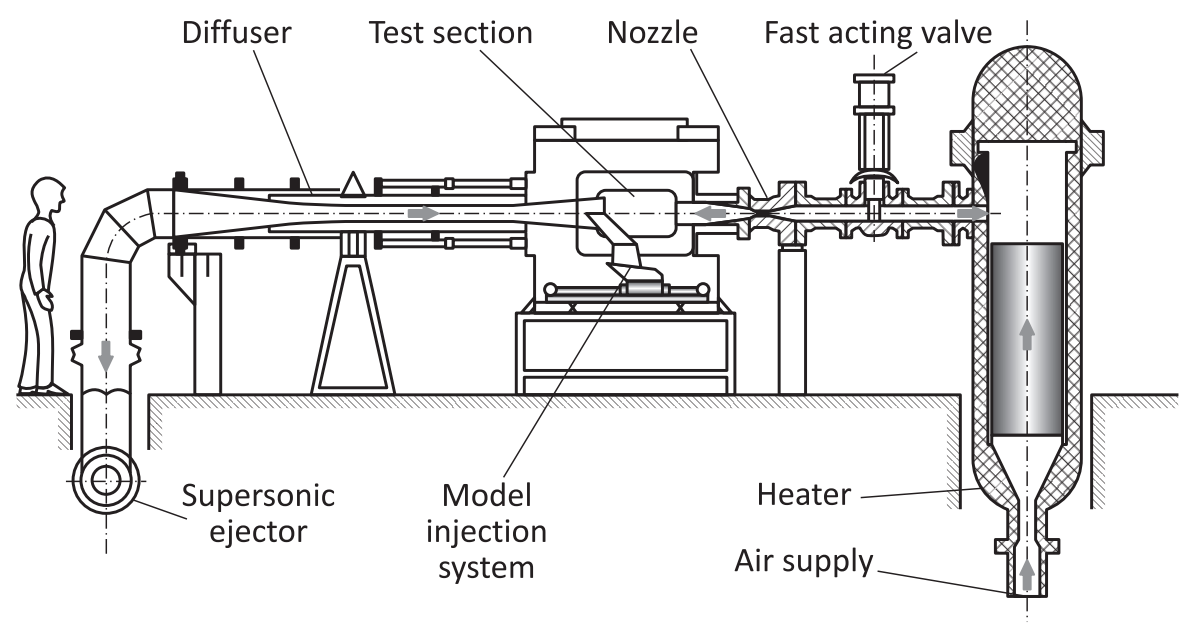

Figure 2 The VKI-H3 hypersonic wind tunnel facility 


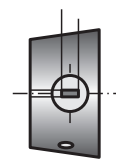

Rectangular streamwise

(the 'spanwise'

is the same

rotated $90^{\circ}$

about its axis)

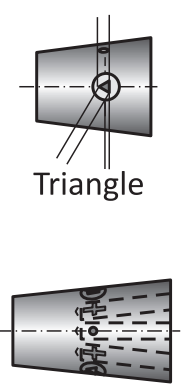

Circular

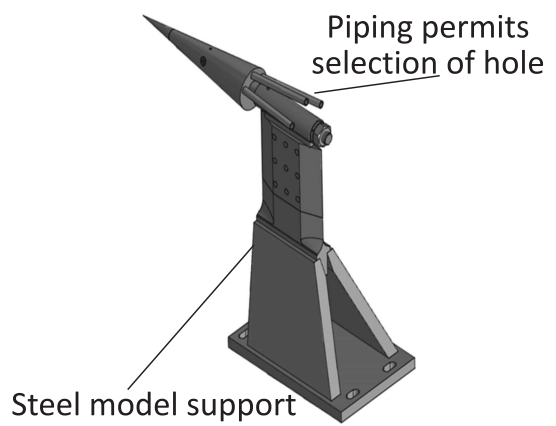

Figure 3 The cone model equipped with the holes of various geometry to inject water jet

optical shadow and Schlieren systems and various other classical measurements techniques are commonly used.

In order to define the test conditions in every run, the total pressure and temperature in the settling chamber are measured using an absolute Statham Strain Gauge pressure transducer and a type K thermocouple. These two values, in conjunction with the nominal test section Mach number (6.0), are adequate to calculate the flow properties such as velocity, Reynolds number, etc. by isentropic flow relations. The minimum stagnation temperature necessary to avoid condensation in the test section is recommended to be $470 \mathrm{~K}$.

The wind tunnel models a cone with 7 degree half-angle. This geometry is an internationally accepted standard test case for modeling a slender axisymmetric body in hypersonic conditions. The cone was equipped with four holes of different geometry in order to investigate the effect of the shape of the issue of the water jet. Each hole was equipped with its own supply tube and the main water supply was connected only to the investigated hole. The model can be seen in Fig. 3 . The holes had the same cross section of $1.5708 \mathrm{~mm}^{2}$.

The typical shape of the holes was: circular, triangle (equilateral), slit aligned streamwise with its long edge, and slit aligned perpendicular to the flow with its long edge. The aim of the variation of the hole shapes was to determine how sharp edges or surfaces with large extension in a given single direction influence the atomization of the water jet.

\section{DESCRIPTION OF THE PHENOMENON}

\subsection{Flat Plate Model}

The breakup of the liquid jet issued from a flat plate surface exposed to Mach 6 hypersonic flow was investigated in detail in [10] from where Fig. 4 was taken to 


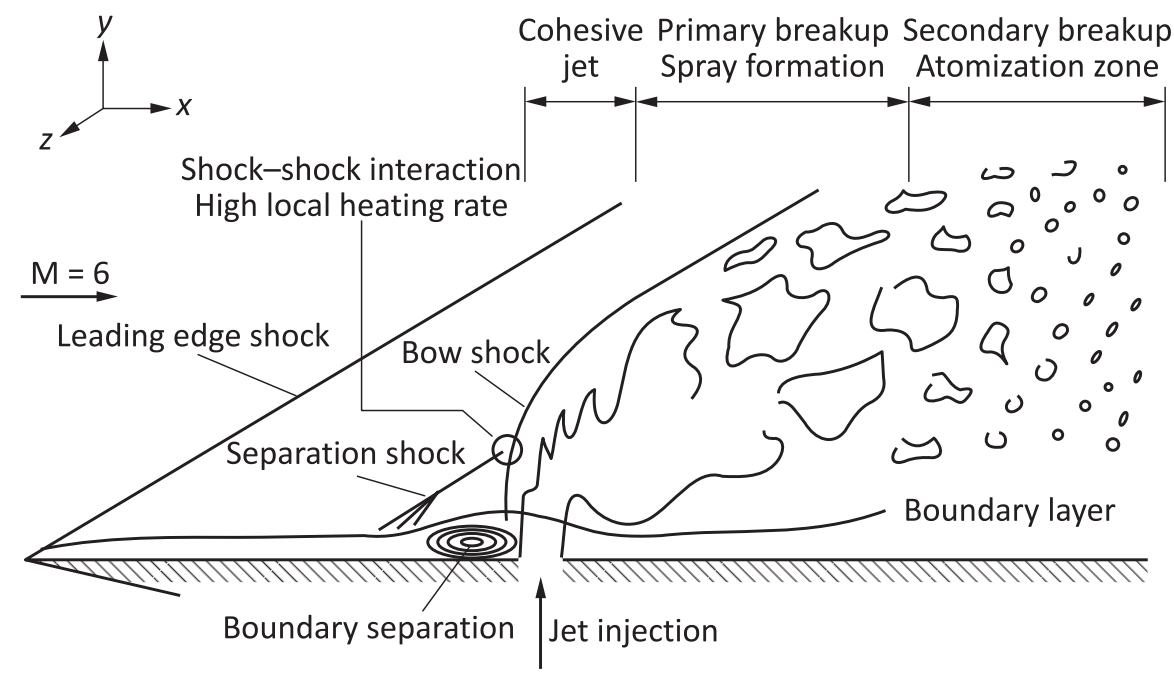

(a)

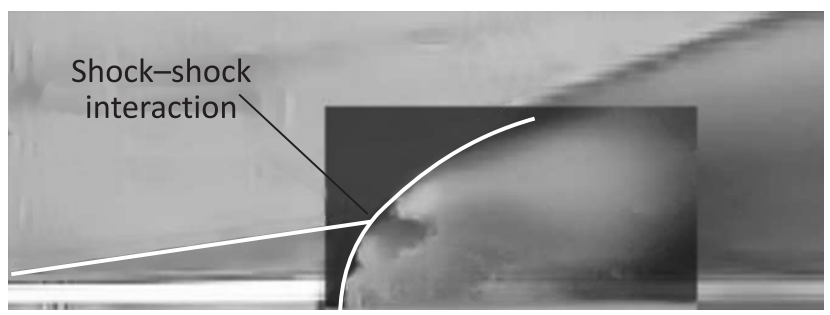

(b)

Figure 4 Liquid jet injected in hypersonic cross flow over a flat plate model [10]: (a) schematic representation; and (b) combination of Schlieren and front lighting droplet cloud measurements for flat plate

represent schematically the process that takes place in this situation. The water jet enters into the hypersonic flow and penetrates at a certain extent depending on the momentum ratio of the incoming hypersonic gas flow and the injected water flow. The water jet is fragmenting into ligaments and droplets forming a plume. The plume occupies a certain volume inside the flow and it interacts with the gas flow. As a result of interaction, a bow shock wave is formed in front of the plume that leads to a significant increase of the pressure and deceleration of the flow near the injection location. Upstream the bow shock wave, the information is propagating upstream inside the boundary layer that leads to its thickening and separation. An oblique shock wave forms due to the presence of the separation zone and it impinges into the bow shock wave. The interaction of 
this intersection of the bow shock and the separation shock plays an important role in the formation of the droplet plume (see Fig. $4 b$ ).

The shape of the plume is three-dimensional; however, there is no detail available on the gas flow field, only in quasi-two-dimensional.

\subsection{Cone Model with Gas Injection}

The gas flow field was visualized by using Schlieren imaging at the H3 wind tunnel. In Fig. 5, it can be seen that the jet introduced perpendicularly to the cone surface leads to a very similar flow field as in the case of a flat plate geometry depicted in Fig. 4. One can observe the thickening of the laminar boundary layer

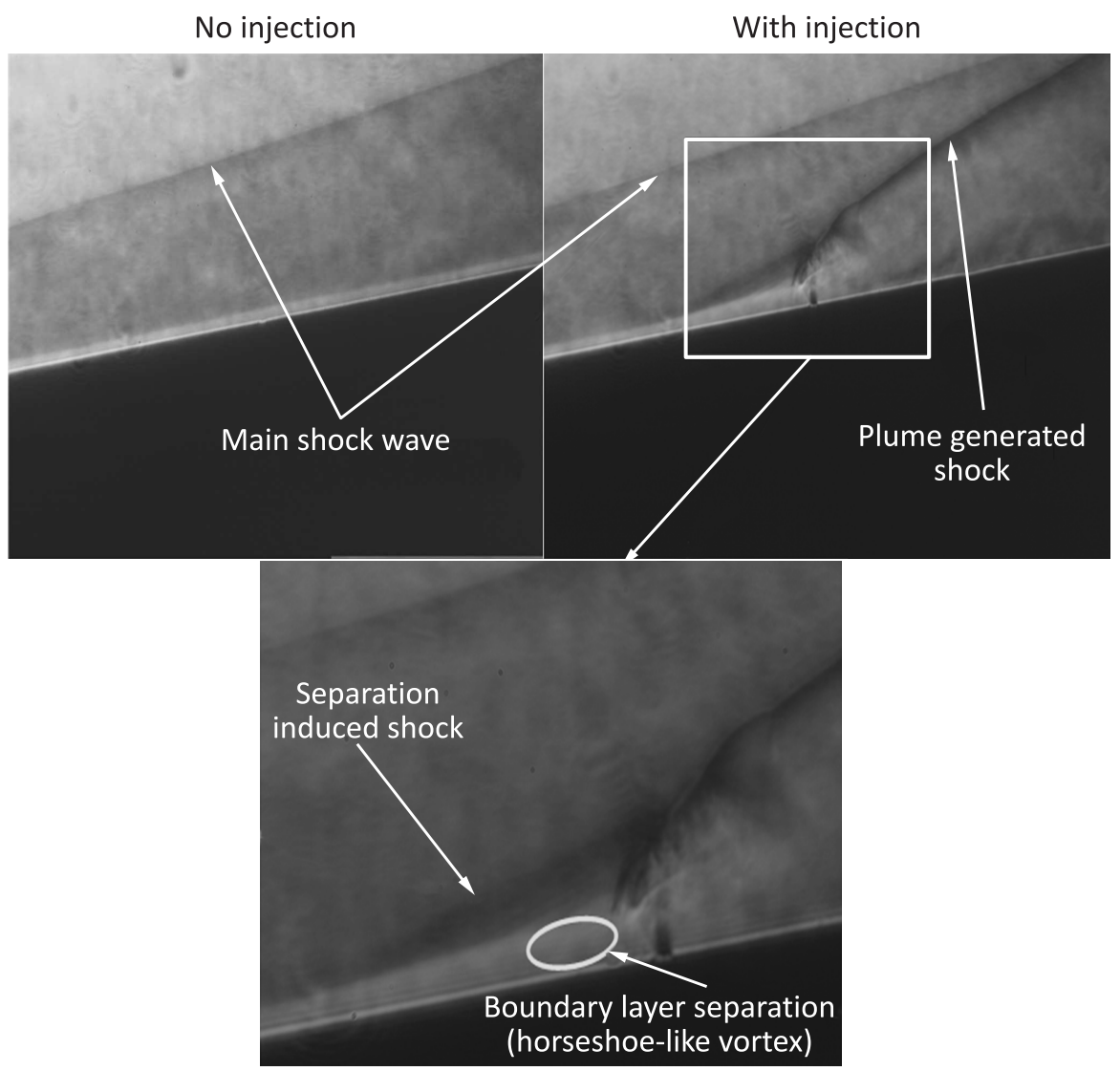

Figure 5 Schlieren imaging of the gas flow field with and without the presence of a gas jet issued from the surface of the cone model. Flow from left to right 
in front of the bow shock. A separation induced shock is also present, but the separation zone, i. e., a recirculation flow region, cannot be identified based on these images. These Schlieren images were obtained by using a 10-nanosecond duration pulsed laser light source; thus, the unsteady turbulent nature of the close vicinity of the jet can be clearly seen. It is also indicated that the incoming boundary layer is fully laminar.

\section{LASER SHEET VISUALIZATION OF THE WATER JET IN HYPERSONIC CROSS FLOW}

In order to understand what processes take place when a water jet is injected in a hypersonic cross flow, flow visualization is needed. Based on the results of the visualization, the type of primary and secondary breakup can be determined.

\subsection{Experimental Setup}

The test chamber of the $\mathrm{H} 3$ wind tunnel is equipped with three windows: two on the sides and one on the top of the chamber. The side windows permit optical access to the camera and the top window gives access to a laser sheet. The setup is represented in Fig. 6. The cone model is placed in the test section of the wind tunnel. An Nd :YAG laser providing 200-millijoule energy pulses with a wavelength of $532 \mathrm{~nm}$ and pulse width of $9 \mathrm{~ns}$ was placed on the top of the test chamber inside a sand bed. The sand bed serves for damping any vibrations of the wind tunnel. The laser beam was shot into a right angle prism (also mounted on the top of the wind tunnel) that directed the beam vertically downwards towards the test section. Below the prism, a cylindrical lens was placed that opened the laser beam into a laser sheet. In order to produce a 1-millimeter thick laser sheet, a focusing lens with 950-millimeter focal length was placed below the cylindrical lens.

The cone model was painted mat black in the region of the visualization in order to avoid reflections of the laser light that perturbs the image on the camera.

In Fig. 6, one can observe the rotameter that was used to measure the flow rate of the water supplied into the wind tunnel model. This way, the injected water flow rate was directly measured.

The water jet without the presence of the gas flow was visualized by front lighting and laser sheet lighting in order to determine its initial structure (Fig. 7). It can be observed that the water jet is contiguous in the beginning but already turbulent. The jet starts to disintegrate into large droplets relatively far (more than $30 \mathrm{~mm}$ ) from the cone surface. It means that when the gas flow is present, then the interaction is taking place between the contiguous water mass and the high-speed gas flow and not droplets and gas flow. 

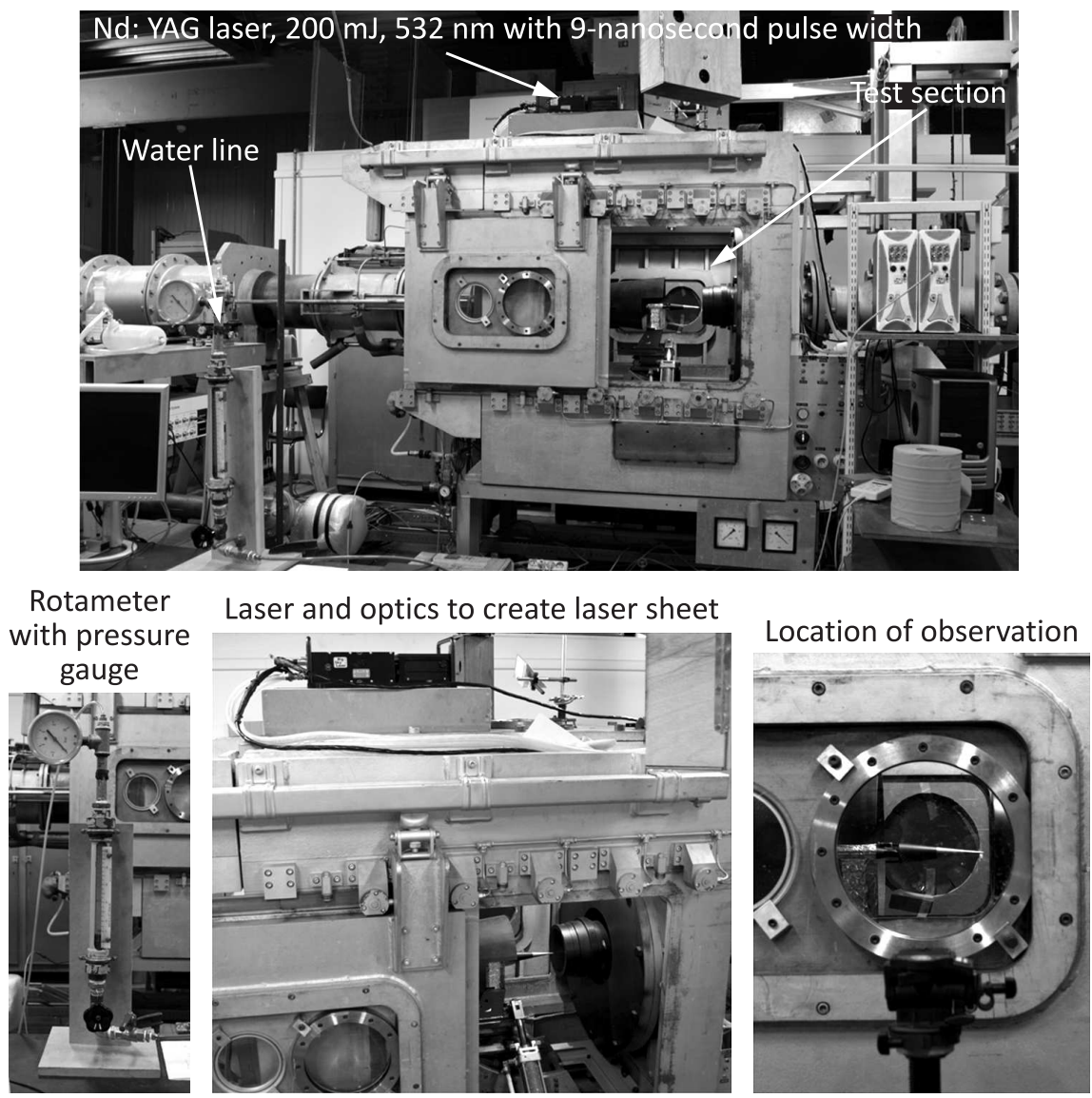

Figure 6 Experimental setup for the laser sheet visualizations

The visualizations of the plume were carried out using a vertical laser sheet aligned with the flow direction. Water was injected upwards normal to the cone surface. The camera was a Nikon D3200 24 megapixel resolution reflex camera equipped with a Nikon Micro-Nikkor $105 \mathrm{~mm} f \#=2.8$ lens set to $f \#=22$ for the present visualizations. The laser was operated at 50-millijoule energy as it was sufficient for a good quality image on the camera sensor.

\subsection{Structure of the Plume}

The plume structure was investigated in case of the 4 different shaped holes at a fixed reservoir pressure of the gas at 20 bar and stagnation temperature 


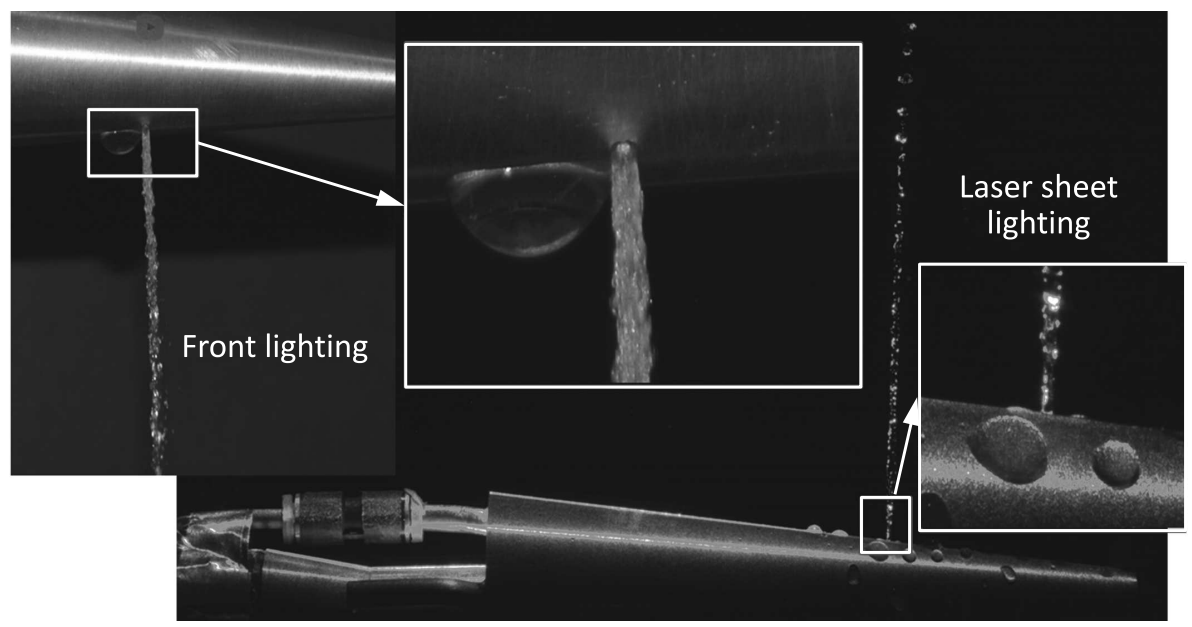

Figure 7 The injected water jet through the circular hole without the presence of the gas flow

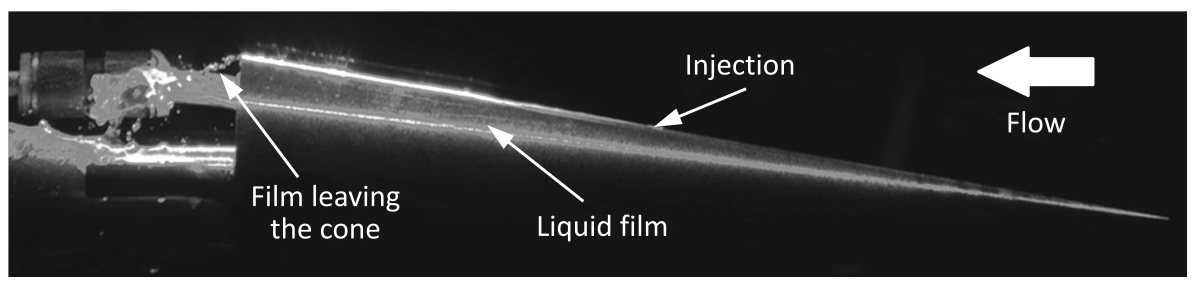

(a)

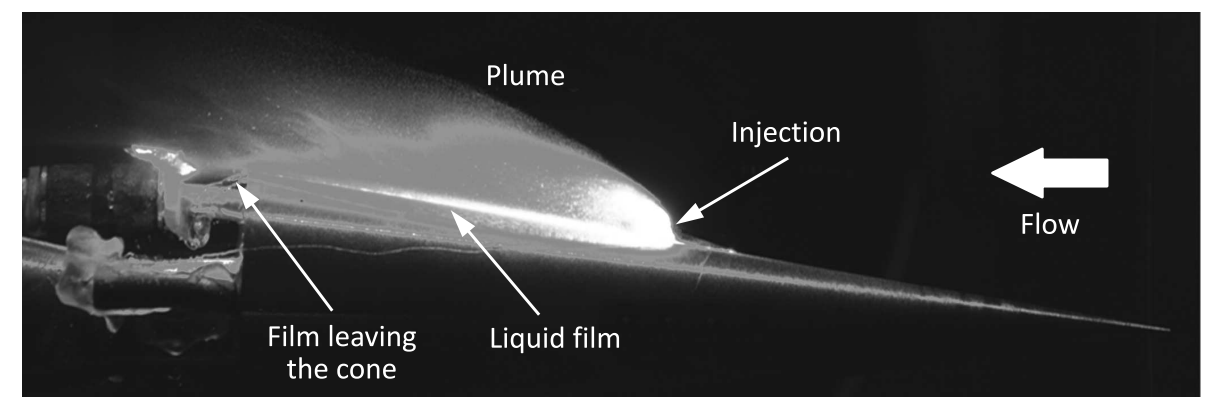

(b)

Figure 8 Water plume structure for water flow rate below $(a)$ and above $(b) 5.46 \mathrm{~g} / \mathrm{s}$ : (a) $\operatorname{Re}_{L}<\sim 6900$, no penetration, liquid flows along cone surface and leaves it at the end of the cone; and $(b) \operatorname{Re}_{L}>\sim 6900$, penetration and atomization 
of $500 \mathrm{~K}$. The injected water flow rate was set to three values: 5.46, 8.67, and $11.89 \mathrm{~g} / \mathrm{s}$ that belongs to water Reynolds number of 6954, 11048 , and 15143 . The Mach number was 6 and constant.

Qualitatively, the flow field under the minimal water flow rate of $5.46 \mathrm{~g} / \mathrm{s}$ and above this value is fundamentally changed as can be seen in Fig. 8. It can be seen that in case of low flow rates, the water jet cannot penetrate into the gas flow. A film of water forms along the cone surface that is expanding in a triangle shaped region until reaching the base of the cone. One can observe the water film leaving the cone downstream its base.
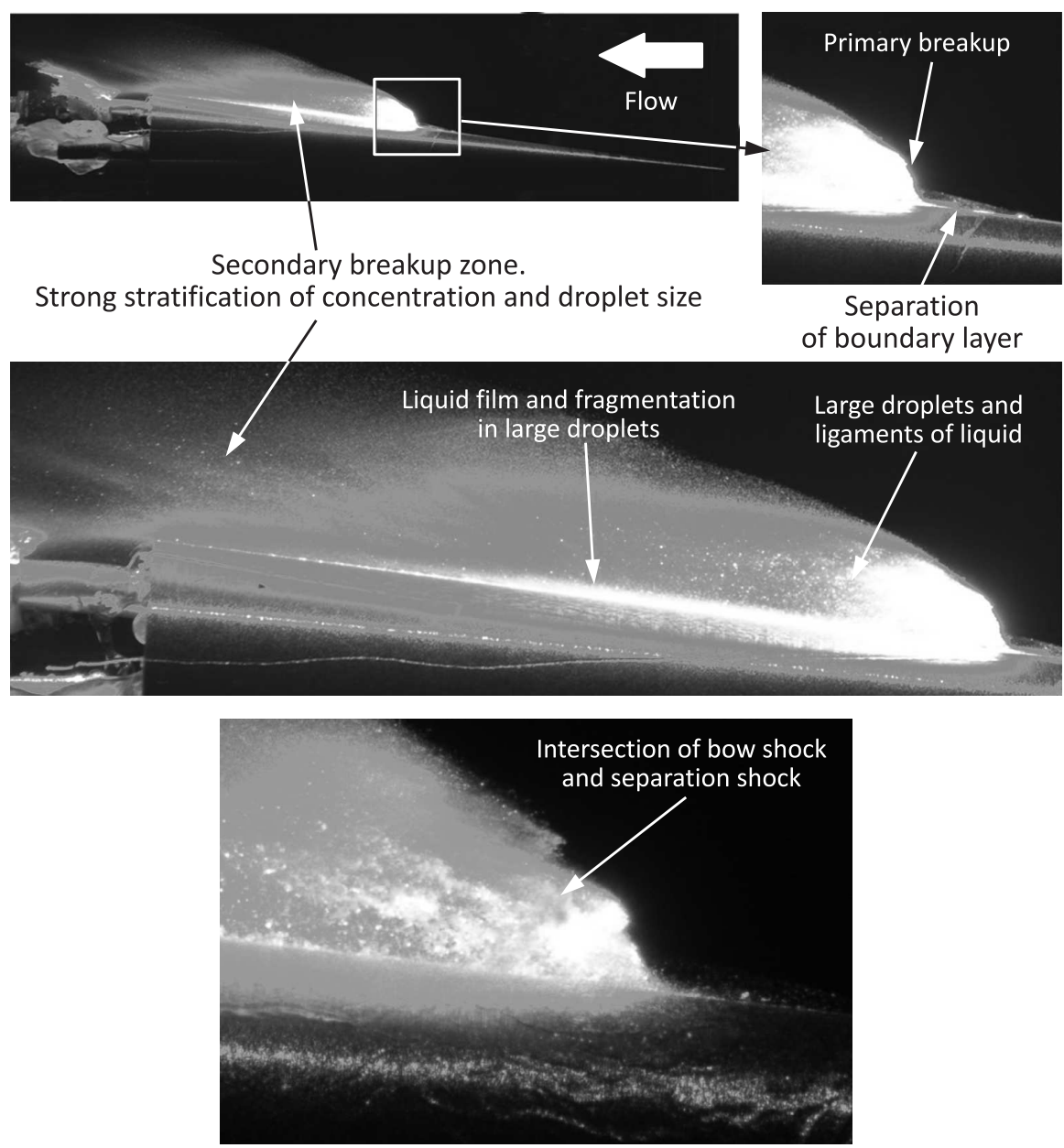

Figure 9 Details of the structure of the water plume in a Mach 6 cross flow. Flow from right to left 
When the flow rate is increased above $5.46 \mathrm{~g} / \mathrm{s}$, the jet is able to penetrate into the gas flow and a large plume forms with a mixture of water ligaments, droplets, and a fragmenting film on the cone surface.

The structure of the plume is analyzed in Fig. 9. It can be seen that the primary breakup takes place in the region of the contiguous column of the injected jet. The front is straight and almost vertical. It can be observed that the droplets are present upstream the injection location which is a clear evidence of the presence of a boundary layer separation with recirculation zone.

The structure of the plume can be decomposed into three characteristic zones.

The first zone is the primary breakup region where the injected jet encounters the Mach 6 cross flow. Here, the body of the liquid jet is 'exploded' by the momentum of the high speed gas flow.

Large liquid ligaments form that break down into small droplets in a very short distance from the injection location. The second zone is the secondary breakup zone that is the region downstream and far away from the wall of the cone, the droplets generated from the liquid jet body fly into this region.

The third zone is near-wall region along the cone downstream the injection location. In this near-wall region, the liquid film is interacting with the impacting small droplets generated close to the injection location. These impacting droplets lead to a fragmentation of the liquid film and, so, to the generation of another class of droplets.

At the downstream part of the plume, one can observe the mixture of various sized droplets: there is the fine fraction at the outer border of the plume; there is a larger droplet size fraction in the central third of the plume; and there is a large droplet fraction close to the wall of the cone.

The liquid film that flows along the cone may also fragment into ligaments after leaving the trailing edge of the cone (Fig. 10). These large ligaments may be present in the wake of the body.

One can also observe that the liquid film is wavy and it is not fragmenting along the surface of the cone. The thin droplet sheet is generated from the breakup of the penetrating part of the jet at this low flow rate case in Fig. 10.

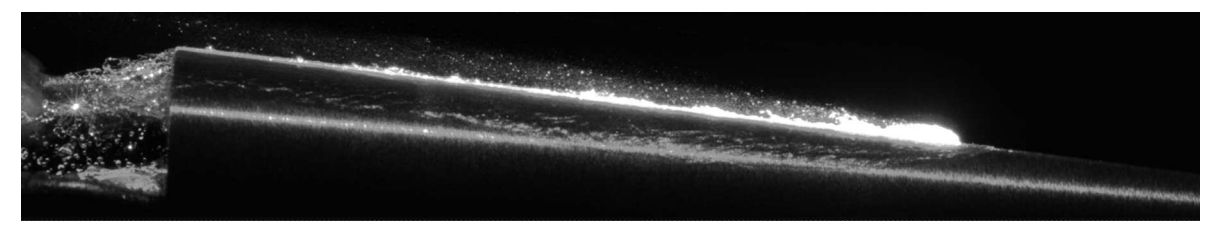

Figure 10 Liquid film fragmenting downstream the trailing edge of the cone into large ligaments of liquid. Flow from right to left 


\subsection{Penetration and Contour of the Plume for Various Hole Geometries}

The same water flow rates were set for the 4 different hole shapes on the cone model in order to see what is the influence of the hole shape on the plume structure. The results can be seen in Fig. 11. The contour images are representing the pixel gray level values in an RGB scale. The images are computed as the average of 5 pictures taken during each test.

The penetration height and the contour of the plumes in case of the various inlet shapes are similar to each other. There are slight differences in the primary breakup front where the jet encounters the Mach 6 flow first after the injection. The differences seem to be less present for the highest flow rate.

Downstream, close to the trailing edge of the cone model, the plume structures are practically the same for all flow rates and all hole shapes. Taking into account that the purpose of the present project is to measure the resultant droplet cloud far downstream the injection point, the shape of the hole has no importance.

The droplet sizing attempts were then carried out using the circular hole geometry.

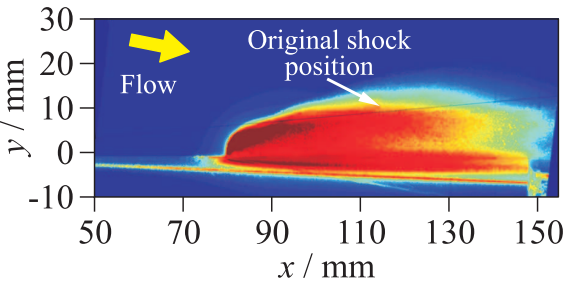

(a)

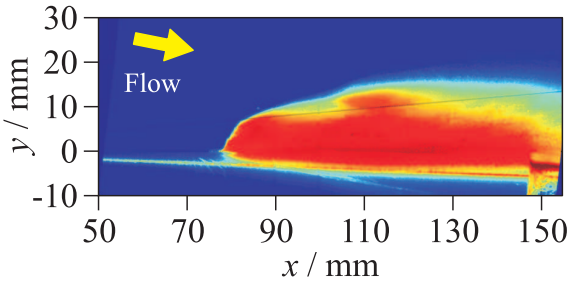

(c)

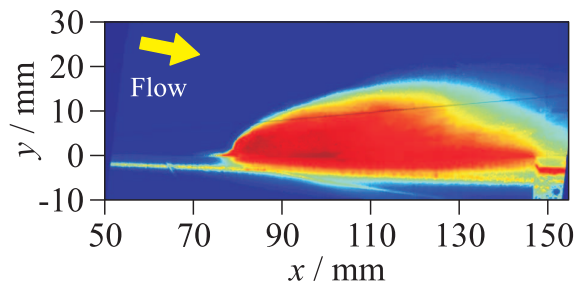

(b)

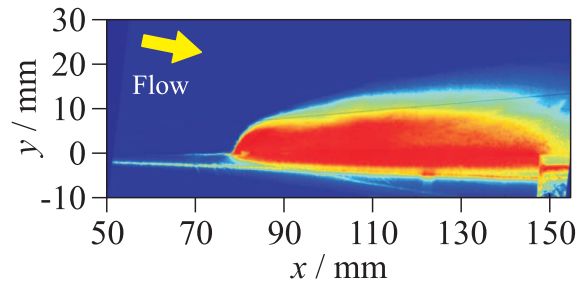

(d)

Figure 11 Jet penetration and plume contour for flow rate $11.89 \mathrm{~g} / \mathrm{s}$. Cone surface is set to horizontal on the images. Contour coloring is proportional to droplet concentration: ( $a$ ) circular hole; $(b)$ rectangular (long edge spanwise) hole; $(c)$ rectangular (long edge streamwise) hole; and $(d)$ triangle hole 


\section{INFRARED LIGHT EXTINCTION SPECTROSCOPY MEASUREMENTS}

Based on preliminary ILIDS measurements, it turned out that the droplets are smaller than $16 \mu \mathrm{m}$. In this size regime, the IR-LES was found to be a promising candidate to measure the droplet size distribution. The IR-LES technique is not sensitive to the high concentration of droplets and it can theoretically measure droplet size distributions below approximately $25 \mu \mathrm{m}$. This upper limit is only theoretical and research is still in progress in order to determine the
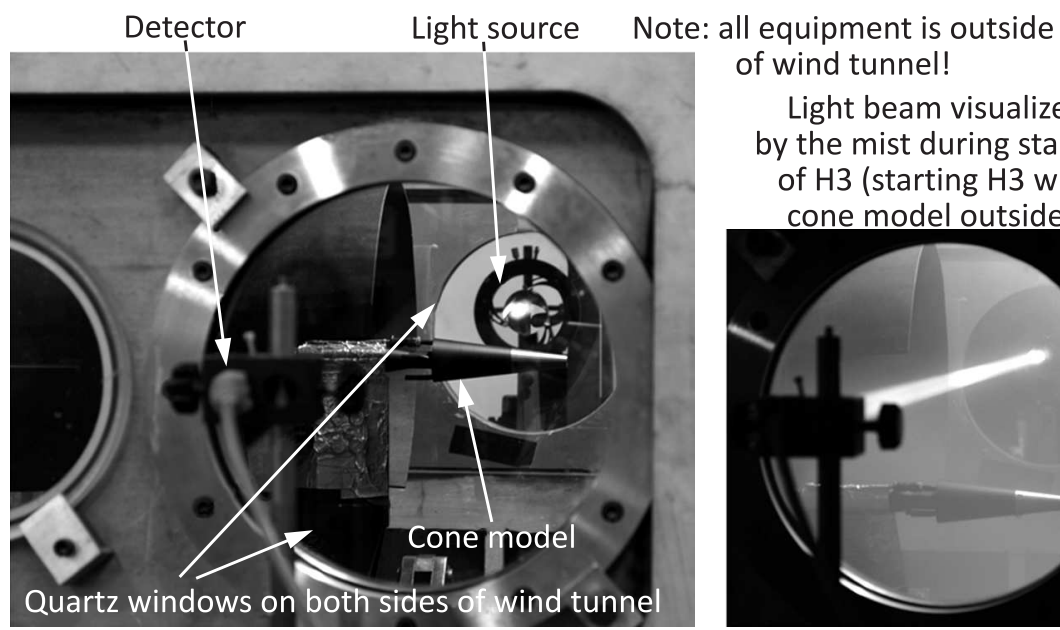
of wind tunnel!

Light beam visualized by the mist during startup of H3 (starting H3 with cone model outside!)
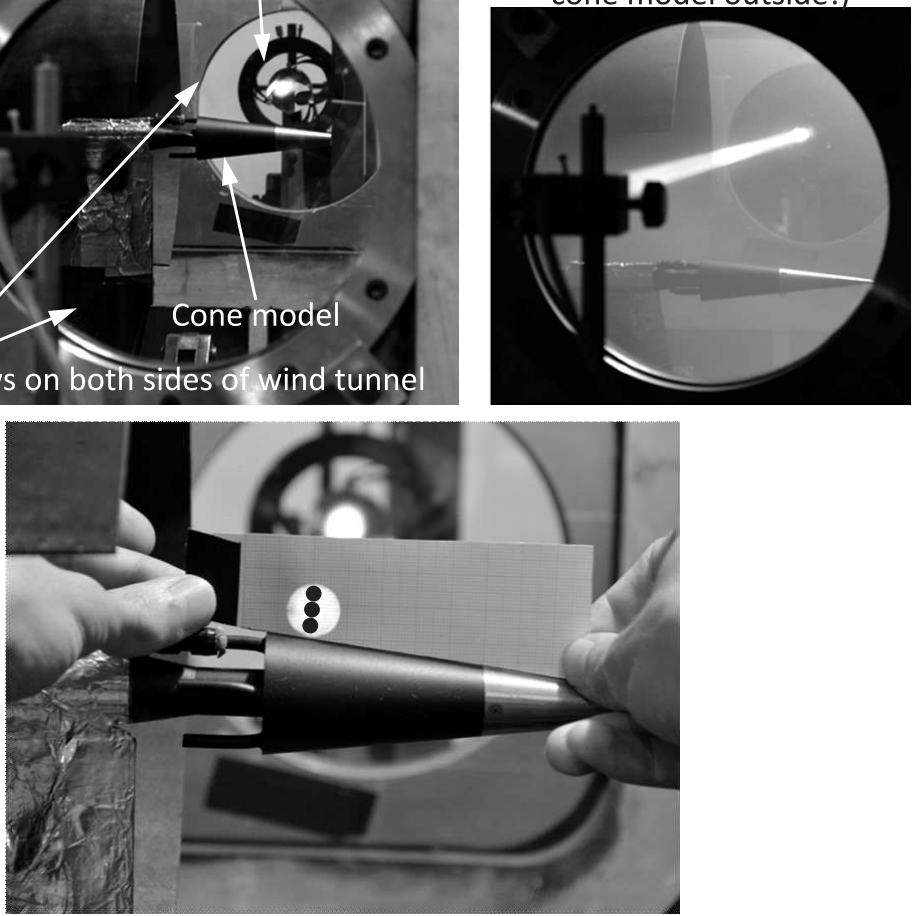

Figure 12 Experimental setup and measurement points for IR-LES technique. Measurement points are indicated on the lower figure with three dots in the light spot (lowest is referred as 'down,' the middle one as 'mid,' and the upper one as 'up' in the paper) 
practical upper size limit that can be reliable determined with this technique. The measurable particle size range depends on the spectral range of the illumination. For example, at nanometer-size range, this technique has already proven to be accurate (LES technique) when using an ultraviolet-near-infrared (UV-NIR) light source. There is also evidence that it reliably and precisely can measure 2-micron-sized droplets/particles. The current case seems to fit well in the applicable size range of this technique. The measurement setup can be seen in Fig. 12.

The setup of the IR-LES measurements is technically simple: an infrared light source has to be placed on one side of the wind tunnel section and a detector has to be put on the other side oriented towards the light source. The detector is connected to a NIR spectrometer (Ocean optics). The light beam and the detectors can be seen in Fig. 13.

On the right side of Fig. 12, the probe volume of the IR-LES can be seen thanks to the appearance of mist during the depressurization of the test chamber before starting the tunnel. It indicates also that this technique is suffering from integration along the line of sight. In the present project, this drawback is an advantage because here, there is not a single point but a small volume of the plume is aimed to be characterized.

It has to be noted that the detector cannot see the full diameter of the light beam but only a 3-millimeter-diameter section of it. The investigated domain is shown in Fig. 12. Three points were investigated at different normal distances from the wall of the cone: $3,7.5$, and $12 \mathrm{~mm}$ from the wall surface.

- Note the icing downstream the base of the cone

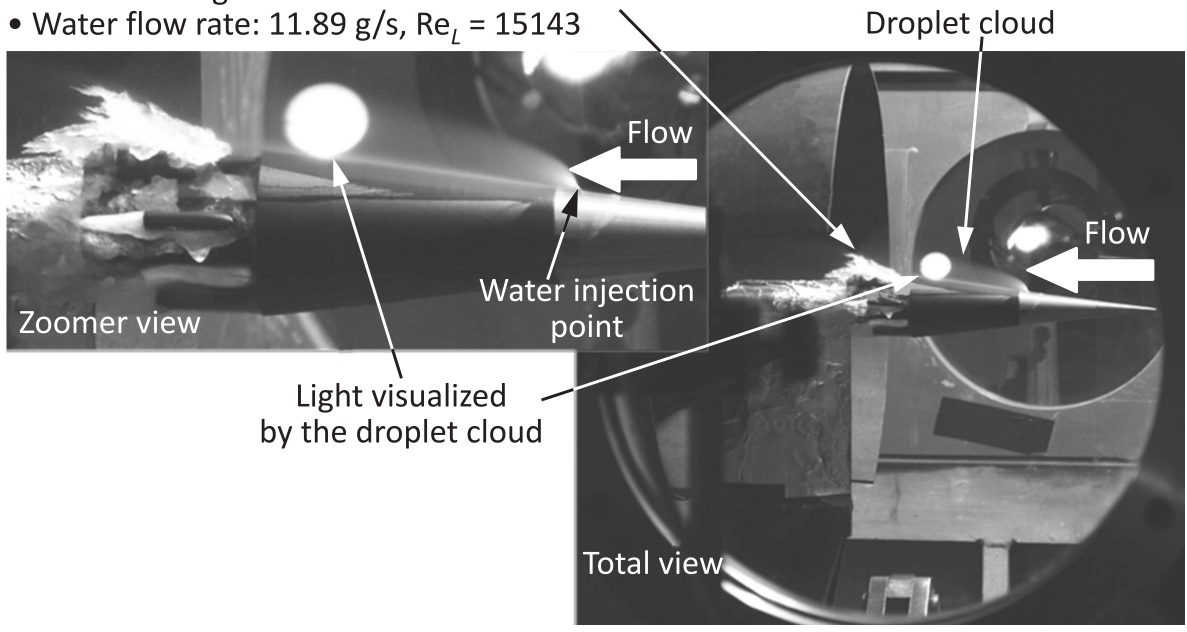

Figure 13 Typical IR-LES measurement for Mach 6 flow over the cone model 
A typical test is shown in Fig. 13 where the water plume is well visible. The water droplets and the liquid film along the cone surface leave the cone at its trailing edge and then form a large ice block during the run.

\subsection{Principle of the Infrared Light Extinction Spectroscopy Technique}

The IR-LES technique is an extension of the traditional LES technique. The only difference is the use of NIR measurement wavelengths. The detailed description of the LES principle together with its experimental validation can be found in $[12]$.

The IR-LES technique uses the measured NIR spectrum $(900-2500 \mathrm{~nm})$ of transmittance $\left(\operatorname{Tr}(\lambda)=I / I_{0}\right)$ for the reconstruction of the particle properties. In the definition of $\operatorname{Tr}(\lambda), I_{0}$ refers to the light intensity incident to the investigated particle cloud and $I$ to the attenuated intensity at the detector side of the particle cloud. The IR-LES technique attempts to find the best fit to a measured transmittance spectrum by means of the modeled one. The fixed parameters of the model depend on the particle shape, morphology, complex refractive index of the particles, and the size parameter $x(x=D \pi / \lambda$ where $D$ is the particle diameter and $\lambda$ is the wavelength of the illuminating light). The variable parameters of the model are the sought particle properties (power spectral density (PSD) and concentration). Assuming the shape and morphology of the particles, the fixed parameters of the model can be a priori calculated from an adequately chosen light scattering theory. When the proper assumptions are used for the calculation of the fixed parameters and a satisfactory fit of the measured and simulated transmittance curves is obtained, the variables of the model should correspond to the underlying particle properties.

According to the Lambert-Beer law,

$$
\operatorname{Tr}(\lambda)=e^{-\tau L}
$$

with $\tau$ being the optical density of the investigated medium and $L$ the path length that the light travels through the particle cloud. The optical density of a particle cloud is a function of the wavelength $(\lambda)$ of the illuminating light and the complex refractive index $(m)$ of the particles. It can be expressed in the following form:

$$
\tau(\lambda, m)=\int_{0}^{\infty} Q_{\mathrm{ext}}(D, \lambda, m) \frac{D^{2} \pi}{4} N(D) d D .
$$

Here, $Q_{\text {ext }}(D, \lambda, m)$ is called the extinction efficiency factor which is the function of particle size $(D)$, wavelength $(\lambda)$, and complex refractive index $(m)$. Assuming spherical and homogeneous particles, $Q_{\text {ext }}$ can be calculated using the Mie 
theory. $N(D)$ is the number of particles contained in the $d D$ interval centered around $D$. The integral is taken over all diameters but it is sufficient to integrate from the smallest to the largest particle being present in the measurement volume. Combining Eqs. (1) and (2) and changing to volumetric particle size distribution functions, the following relation is obtained:

$$
-\ln (\operatorname{Tr}(\lambda)) \frac{2}{3 L}=\int_{D_{\min }}^{D_{\max }} Q_{\text {ext }}(D, \lambda, m) \frac{V(D)}{D} d D
$$

The discretized form of Eq. (3) can be obtained using linear quadrature for both $D$ and $\lambda$ leading to a linear algebraic equation:

$$
T=S V
$$

where $T$ is the discretized form of the left-hand side of Eq. (3); $S$ is the matrix related to the extinction efficiency factors; and vector $V$ is the unknown volumetric particle size distribution function. In order to retrieve the volumetric PSD function $V(D)$, Eq. (4) should be inverted. Unfortunately, due to the smoothing effect of matrix $S$, the above system is ill-conditioned and numerically unstable.

In the present study, Eq. (4) is solved by the nonnegative Philips-Twomey regularization, which is optimized by the $L$-curve method. The latter is described in details in $[12,13]$. Such an inversion process should be validated both numerically and experimentally before applying the IR-LES technique as a particle characterization tool.

The numerical validation of the inversion algorithm is not presented here in details. Instead, only one representative example is shown. A solid particle aerosol consisting of $\mathrm{KCl}$ (Potassium Chloride) particles have been modeled with a lognormal particle size distribution function with mean diameter $200 \mathrm{~nm}$ and dispersion factor 0.3 . The transmittance function of this distribution has been modeled on the 200-1000-nanometer interval. The model aerosol exhibited a minimal transmittance of 0.89 at the wavelength $228 \mathrm{~nm}$. The modeled transmittance spectrum has been disturbed by normally distributed random error with amplitude $1 \%$ of the actual transmittance value. The inversion of the hypothetical data has been performed for 50 different random errors. The mean value of the results has been calculated at each diameter. The comparison of the generated and the retrieved mean distribution functions is shown in Fig. 14. The comparison displays the satisfactory reconstruction of the hypothetic PSD function. The peak of the function is slightly underestimated but the mean value and standard deviation are well captured.

The experimental validation of the technique is presented in details in [12]. Validation measurements have been performed for both monodisperse and polydisperse particle size distribution functions in the submicron size range. The 


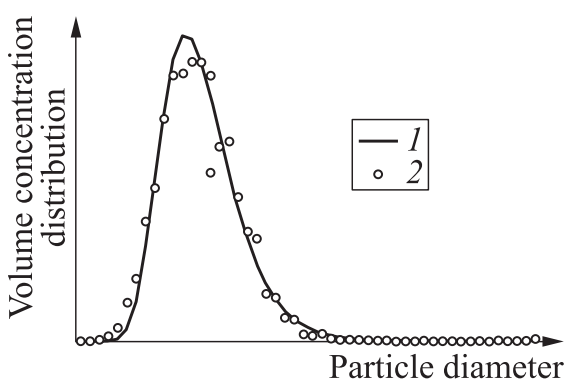

Figure 14 Comparison of modeled (1) and retrieved (2) particle size distribution functions

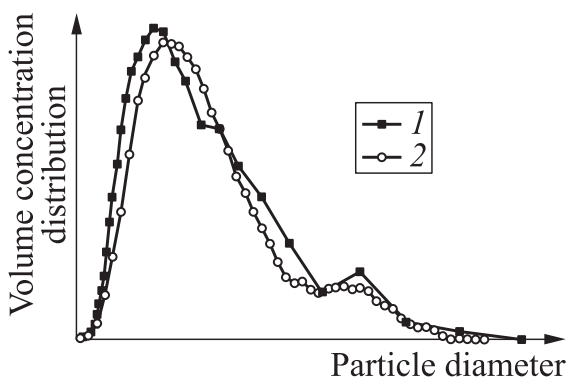

Figure 15 Comparison of size distributions measured with the SMPS (1) and the LES (2) techniques. The test aerosol is a $\mathrm{KCl}$ solid particle aerosol

technique was found to be capable of providing the measured values within 5 percent accuracy compared to the reference values. Since the present liquid breakup process is expected to produce a polydisperse size distribution, here only a polydisperse validation case is shown. $\mathrm{A} \mathrm{KCl}$ solid particle aerosol has been produced by means of atomizing and drying a precursor aqueous $\mathrm{KCl}$ solution (1 percent mass concentration). The produced aerosol has been analyzed by a reference method: Scanning Mobility Particle Sizer with Condensation Particle Counter (SMPS-CPC) of Grimm Aerosol GmbH (SMPS 5.416) which is capable of measuring particle sizes in the range 1-1000 nm. The same aerosol has been measured online with the LES technique. The comparison of the obtained distributions is presented in Fig. 15. A satisfactory agreement is displayed in terms of mean particle size, standard deviation, and concentration. It is noted that the small secondary peak centered at $700 \mathrm{~nm}$ is equally identified by both measurement techniques, confirming that the LES technique is able to provide detailed size distribution information.

\subsection{Results with Infrared Light Extinction Spectroscopy Technique}

\subsubsection{Test conditions}

On the side of IR-LES measurements, the following settings were applied: wavelength range - 900-2500 nm; exposition time of single spectrum - $10 \mathrm{~ms}$; spectra to average -20 ; acquisition period $-220 \mathrm{~ms}$; acquisition duration $-5 \mathrm{~s}$; and number of acquisitions per test -22 . The tests were carried out for three upstream unit Reynolds numbers and one flow rate of water, $9.2 \mathrm{mln} / \mathrm{m}$ (referred as 10-bar reservoir pressure), $17.7 \mathrm{mln} / \mathrm{m}$ (20 bar), and $26 \mathrm{mln} / \mathrm{m}$ (30 bar). The Reynolds number was set by the reservoir pressure of the wind tunnel. 
As the wind tunnel startup and the water injection take time and need careful manipulation, the series of acquisitions had to be checked in order to find the operation point of the system that can be treated as stabilized and constant. This investigation was done based on the transmittance level.

\subsubsection{Droplet size distributions}

The volumetric droplet size distributions are shown in Fig. 16. It can be seen that most of the volumetric contribution $(\sim 80 \%)$ can be found below a size
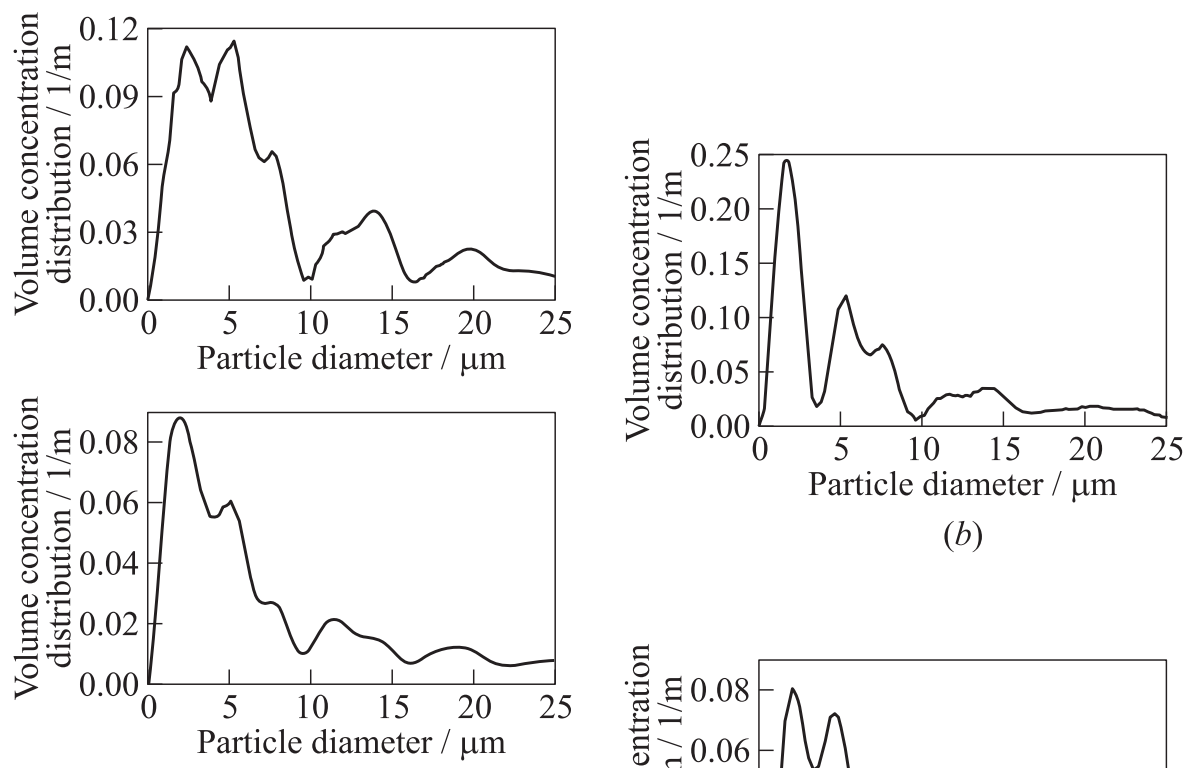

(b)

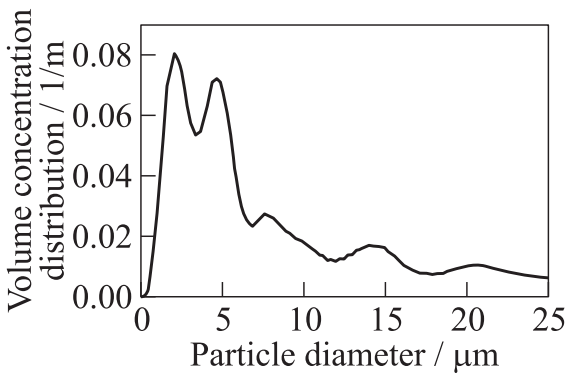

(c)

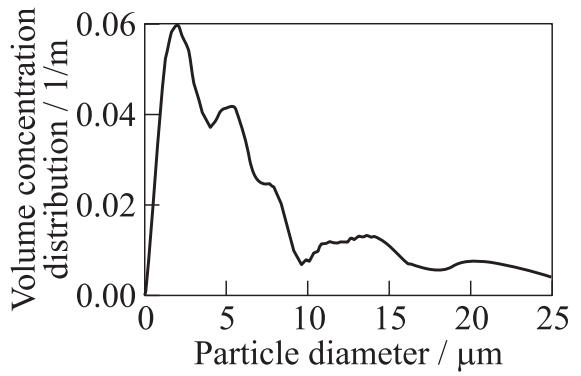

(a)

Figure 16 Volumetric droplet size distributions for various pressures and locations of the detector: $(a)$ middle point of measurement spot (upper frame - 10 bar; middle 20 ; and lower frame - $30 \mathrm{bar})$; and $(b)$ upper and $(c)$ lower points of measurements spot at 20 bar 
of $15 \mu \mathrm{m}$. The percentage of the number of droplets below $15 \mu \mathrm{m}$ would be even larger. This agrees well with the ILIDS investigation, which has concluded that the droplets under investigation should be smaller than $16 \mu \mathrm{m}$, since no interference fringes were detected.

One can observe that the majority of the droplets are in the order of $2 \mu \mathrm{m}$ : the main peak can be found at this size in all of the tested cases. The second peak at $5 \mu \mathrm{m}$ is also present in all the measured cases. Some further, less important features can also be observed but these are more uncertain due to approaching the measurement limit of the IR-LES technique. At sizes above $15 \mu \mathrm{m}$, an asymptotic decrease of the droplet size distributions can be observed in all the measured cases. The small volume contribution of these large droplets means that they are very few in number.

It is very likely that the highlighted features of the droplet size distribution functions correspond to different breakup mechanisms. They are present in all the test cases but their relative weights vary. Fixing the pressure at 20 bar and varying the probe position from bottom to top, one may observe how the relative contribution of the peak at $5 \mu \mathrm{m}$ increases. This result suggests that the 2 -micron peak originates from the drops impacting onto the liquid film formed on the cone surface downstream the injection region. Therefore, they can be mostly found close to the cone surface. The 5 -micron peak may be produced from the primary breakup of the liquid jet. As the laser sheet visualization has confirmed, these larger drops are more and more present in the regions further away from the cone.

The effect of pressure can be studied by means of fixing the probe position at the middle of the illuminated spot and varying the pressure between 10, 20,

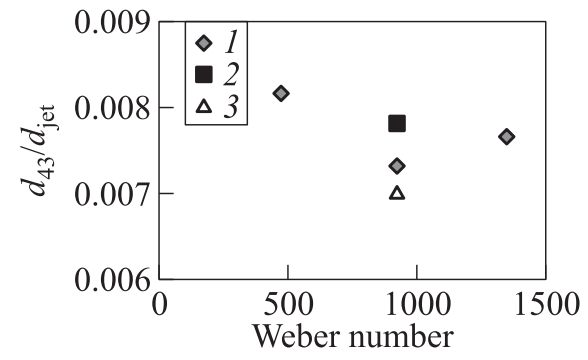

Figure 17 Mean droplet size dimensionalized by the jet diameter as a function of Weber number based on jet diameter: 1 - 1-millimeter hole diameter; 2 1-millimeter hole diameter (repeat of measurements); and $3-2$-millimeter hole diameter and 30 bar. The IR-LES results show that increasing the pressure from 10 to 20 bar results in smaller contribution from the 5-micron peak. However, the tendency does not continue when increasing the pressure to 30 bar. The reason of this effect should be a subject of future investigations.

Dependence of the volume mean diameter on the Weber number can be seen in Fig. 17. The characteristic length that is used to compute the Weber number in this case is the jet diameter as it is fixed by the geometry. With Reynolds number, the density is changing; thus, the Weber number is varying. One can observe that there is a slight decrease in the mean droplet 
diameter with increasing Weber number, but the values are changing within the estimated uncertainty of the measurement. This indicates that as the breakup process is of the catastrophic type, the final droplet size is immediately formed from the erupting ligaments.

\section{CONCLUDING REMARKS}

The present paper summarizes the atomization of a continuous water jet issued through an arbitrary shaped hole on the surface of a 7 degree half-angle cone into hypersonic (Mach 6) flow. Schlieren imaging was used for analyzing the gas flow field in the injection region. It was confirmed that the incoming boundary layer is fully laminar. The main observed feature is a bow shock which forms around the injected plume. Applying Schlieren visualization in the presence of the water jet revealed the segmentation of the water plume on the liquid front where the shock-shock interaction occurs between the separation-induced shock and the bow shock.

The front lighting analysis of the water jet showed that the jet starts to disintegrate into large droplets relatively far from the cone surface. It means that when the gas flow is present, the interaction is taking place between the contiguous water mass and the high-speed gas flow and not droplets and gas flow.

Laser sheet visualization was applied for studying the effect of the flow rate of the injected water jet and the shape of the holes: circular, rectangular, and triangular. It is shown that in case of low flow rates, the water jet cannot penetrate into the gas flow. The shape of the holes play a detectable role in the vicinity of the injection but downstream, at the location of the trailing edge of the cone, the droplet cloud has very similar structure.

The laser sheet visualization of the atomized plume revealed three different regions that result from different types of atomization mechanisms. The first region is where the liquid jet encounters the Mach 6 hypersonic flow; thus, primary breakup takes place. The created ligaments further break up into small drops which fly into the second region. The latter is located downstream the injection point and far away from the cone surface.

A new optical particle characterization method, the IR-LES technique, was applied for determining the droplet size distribution of the plume. The IRLES technique proved feasibility and displayed several advantages such as the relatively easy installation and alignment, fast measurement, and immediate retrieval of droplet size statistics. The IR-LES droplet size distributions contain two main peaks at 2 and $5 \mu \mathrm{m}$. The measurement of droplets larger than $10 \mu \mathrm{m}$ is uncertain, since the maximum size limit of the IR-LES technique is a subject of the ongoing investigations. 
The results show that the mean droplet diameter is practically independent on the Weber number in this range. The breakup is the catastrophic type and thus, from each erupted ligament of liquid, the smallest possible droplet size forms.

\section{REFERENCES}

1. Pilch, M., and C. Erdman. 1987. Use of break-up time data and velocity history data to predict the maximum size of stable fragments for acceleration-induced break-up of a liquid drop. Int. J. Multiph. Flow 13:741-757.

2. Unal, A. 1987. Effect of processing variables on particle size in gas atomization of rapidly solidified aluminium powders. Mater. Sci. Technol. 3:1029-1039.

3. Karagozian, A.R., K. S.C. Wang, and O. I. Smith. 2003. Jets injected normally into compressible crossflow. Eds. A. R. Karagozian, L. Cortellezzi, and A. Soldati. Wien-New York, NY: Springer. 25-38.

4. Kolpin, M., K. Horn, and R. Reichenbach. 1968. Study of penetration of a liquid injectant into a supersonic flow. AIAA J. 6:853-858.

5. Catton, I., D. Hill, and R. McRae. 1968. Study of liquid jet penetration in a hypersonic stream. AIAA J. 6:2084-2089.

6. Joshi, B. P., and J.A. Schetz. 1975. Effect of injector shape on penetration and spread of liquid jets. AIAA J. 13:1137-1138.

7. Nejad, A., and J. Schetz. 1984. Effects of viscosity and surface tension on a jet plume in supersonic crossflow. AIAA J. 22:458-459.

8. Lin, K., P. Kennedy, and T. Jackson. 2002. Penetration heights of liquid jets in high-speed crossflows. 40th AIAA Aerospace Sciences Meeting and Exhibit. Reno, NV, USA.

9. Srulijes, J., and F. Seiler. 2009. Shock tube experiments at ISL on liquid fragmentation. Liquid fragmentation in high speed flow. Eds. P. Rambaud and C. Asma. VKI lecture ser. Von Karman Institute for Fluid Dynamics. Vol. 2009-04.

10. Asma, C. O. 2009. Experimental investigation of liquid fragmentation in hypersonic cross flow. University of Gent. PhD Thesis.

11. Adler, C. 2013. Liquid fragmentation phenomena in hypersonic flows. VKI Report VKI 2013-01.

12. Horvath, I. T., P. Colinet, and M. R. Vetrano. 2016. Assessment of the light extinction spectroscopy technique for submicron particle characterization. Powder Technol. 291:375-382.

13. Hansen, P. C. 1998. Rank-deficient and discrete ill-posed problems: Numerical aspects of linear inversion. SIAM. $264 \mathrm{p}$. 